\title{
VISCOMETER UTILIZING A FLOATING CHARGED MAGNETIC PARTICLE
}

\author{
K. GLOOS, J.H. KOIVUNIEMI, W. SCHOEPE\#, J.T. SIMOLA, and J.T. TUORINIEMI
}

Low Temperature Laboratory, Helsinki University of Technology, 02150 Espoo, Finland*

\begin{abstract}
A new type of high sensitivity viscometer is presented, reaching Q-values of up to one million at $0.3 \mathrm{kHz}$ resonance frequency. Possible applications to thermometry and for the creation and detection of vortex rings in superfluid helium are discussed.
\end{abstract}

\section{INTRODUCTION}

Typical low temperature viscometers, like vibrating wires and torsional oscillators, have a sensor which is mechanically connected to the surroundings. This introduces some parasitic damping. By using a floating magnetic particle, levitated by the Meissner repulsion between two superconducting plates, this problem can be largely overcome. Because the particle has a small but macroscopic size, it should be possible to create and study vortex rings in superfluid helium with a device of this type.

\section{EXPERIMENTAL}

Our experimental cell (Fig. 1a) is made of niobium. Top and bottom plates act as a capacitor with a gap of about $1 \mathrm{~mm}$. The lower plate contains an electrically insulated $1 \mathrm{~mm}$ diameter $\mathrm{Nb}$ wire as a collector.

The magnetic particle, cut from a $\mathrm{SmCo}_{5}$ permanent magnet, was ground to about $0.1 \mathrm{~mm}$ diameter. The particles that we have investigated are not ideally spherical but have a surface roughness of about $0.01 \mathrm{~mm}$. Above $\mathbf{T}_{\mathfrak{c}}$ of $\mathrm{Nb}$, the capacitor is charged to about $500 \mathrm{~V}$. By this the particle, resting on the bottom plate, is also charged and starts to move between the two capacitor plates as discussed in Ref. 1. From the integrated current sensed by the collector when the particle hits it, one obtains an estimate of the charge on the particle, typically $1 \mathrm{pC}$. When cooling through $\mathrm{T}_{\mathrm{c}}$ the signal vanishes since, owing to the Meissner effect, the charged particle is now floating between the superconducting capacitor plates. Because of the finite charge left on the particle, its motion can still be detected via the ac-current signal, induced on the collector.

The resonance frequency for the vertical motion of the charged particle can be found, e.g., by gently knocking on the device and by Fourier transforming the signal. Over a very wide range the frequency depends linearly on the dc bias volt-

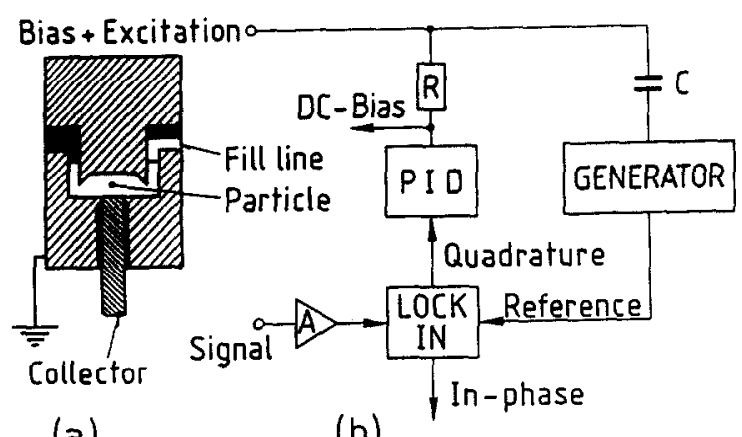

(a)

(b)

FIGURE 1

(a) The experimental cell, made of three $\mathrm{Nb}$ parts which are insulated from each other by Stycast 1266 (black area). (b) The constant-frequency phase-locked loop.

age, which determines the particle's vertical equilibrium position inside the gap. The bias-dependent resonance frequency, with a typical sensitivity of about $50 \mathrm{mHz} / \mathrm{V}$, offers the possibility to drive the particle at constant excitation frequency in a feedback loop, using the cell itself as a voltage controlled oscillator (Fig. 1b). In this mode parasitic frequency-dependent pickup signals can be handled easily.

\section{RESULTS}

To demonstrate the feasibility of our viscometer, the pressure-dependent damping of the particle's movement in ${ }^{4} \mathrm{He}$ gas at $4.2 \mathrm{~K}$ was investigated. Results are shown in Fig. 2. As expected for an ideal gas at high pressures, the quality factor $Q \propto 1 / \sqrt{ } \mathrm{P}$ as long as the viscous penetration depth is smaller than the particle size; at lower pressures, when the mean free path of the $\mathrm{He}$ atoms is larger than the particle size, $\mathrm{Q} \propto 1 / \mathrm{P}$. At $0.1 \mathrm{~Pa}$ the measured $\mathrm{Q}$-value

\footnotetext{
* Work supported by the Academy of Finland and by the Korrber-Stiftung (Hamburg). \# Permanent address: Institut fur Angewandte Physik, Universität, 8400 Regensburg. West Germany.
} 


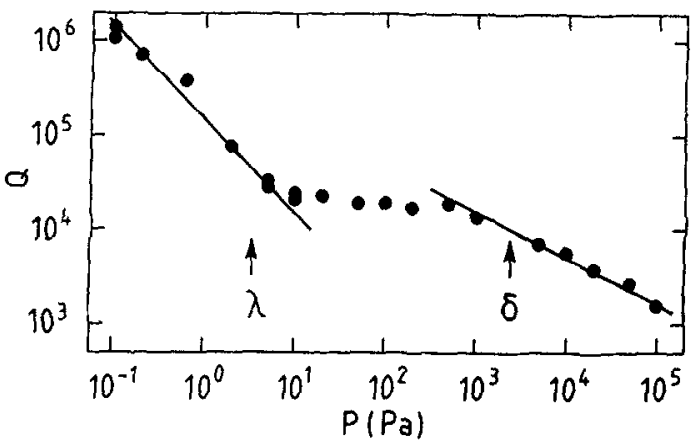

FIGURE 2

Measured Q-value in ${ }^{4} \mathrm{He}$-gas at $4.2 \mathrm{~K}$. The pressure is correct to within a factor of 2 . Above $10 \mathrm{~Pa}, \mathrm{Q}$ was obtained from the linewidth of the resonance, while at lower pressures it was calculated from the exponential decay time of the particle's oscillations. Also indicated is the pressure at which the viscous penetration depth $\delta$ and the mean free path $\lambda$ of $\mathrm{He}$ atoms equal $0.1 \mathrm{~mm}$, the approximate radius of the particle used. Resonance frequency is $299 \mathrm{~Hz}$.

reaches one million. At the resonance frequency of $299 \mathrm{~Hz}$ this corresponds to a resonance width of $0.3 \mathrm{mHz}$ only. The resonance frequency was stable to better than $50 \mathrm{mHz}$ over a period of two weeks.

In this regime, the response of the particle no longer depends linearly on the excitation (Fig. 3). We suspect that this effect is caused by the anharmonicity of the potential well in which the particle moves, also responsible for the bias-dependent resonance frequency.

Another interesting feature is the appearance of a second mode, the signal size of which is indepen. dent of the excitation amplitude. This is very likely caused by rotation of the particle around its mag. netic axis. The electrical dipole moment, estimated from the signal size, is about $10^{-18} \mathrm{Cm}$, and is due to the particle's deviation from spherical symmetry.

\section{POSSIBLE FURTHER APPLICATIONS}

Because of its very low residual damping, the device is ideally suited for studies of low dissipation processes.

We have developed this measuring technique to investigate ${ }^{4} \mathrm{He}$ down to $\mathrm{mK}$-temperatures. Since the phonon density in ${ }^{4} \mathrm{He}$ below $0.5 \mathrm{~K}$ varies strongly with temperature (2), one has a very sensitive thermometer. The same would apply to ${ }^{3} \mathrm{He}$ at $\mu \mathrm{K}$-temperatures (3).

Because the particle has a small mass, the oscillator can resolve very small energy changes, e.g., those due to vortex ring production $(4,5)$.

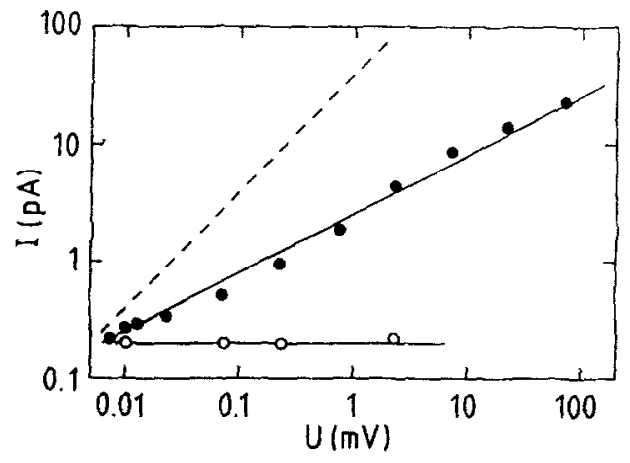

FIGURE 3

Measured RMS current induced by the moving particle, as a function of the excitation voltage at $299 \mathrm{~Hz}$ in ${ }^{4} \mathrm{He}$ gas at $4.2 \mathrm{~K}$ and under a pressure of about $0.1 \mathrm{~Pa} .1 \mathrm{pA}$ corresponds to about $1 \mu \mathrm{m}$ displacement. Solid and open circles refer to translational and rotational modes, respectively. The dashed line is the signal expected for an ideal harmonic oscillator at $Q=10^{6}$, extrapolated from the low-Q response.

Another large area of investigation would be provided by the nonresonant rotational mode. For pure rotation the flow is stationary and the velocity can be chosen at will.

An alternative would be to employ a superconducting particle and replace the superconducting suspension by an active magnet system. The essential advantage of this method would be the possibility to adjust the particle's resonance frequency by simply changing the magnetic field gradient.

\section{ACKNOWLEDGEMENTS}

We gratefully acknowledge discussions with Prof. M. Krusius.

\section{REFERENCES}

(1) J.T. Simola and J.T. Tuoriniemi, this conference

(2) M. Morishita, T. Kuroda, A. Sawada, and T.Satoh, J. Low Temp. Phys. 76 (1989) 387

(3) J.P. Camey, K.F. Coales, A.M. Guenault, G.R. Pickett, and G.F. Spencer, J. Low Temp. Phys. 76 (1989) 417

(4) A.L. Fetter, Vortices and Ions in Helium, in: The Physics of Liquid and Solid Helium, Part I, eds. K.H. Bennemann and J.B. Ketterson, (J. Wiley and Sons, New York, 1976) pp. 207

(5) P.C. Hendry, N.S. Lawson, P.V.E. McClintock, C.D.H. Williams, and R.M. Bowley, Phys. Rev. Lett. 60 (1988) 604 